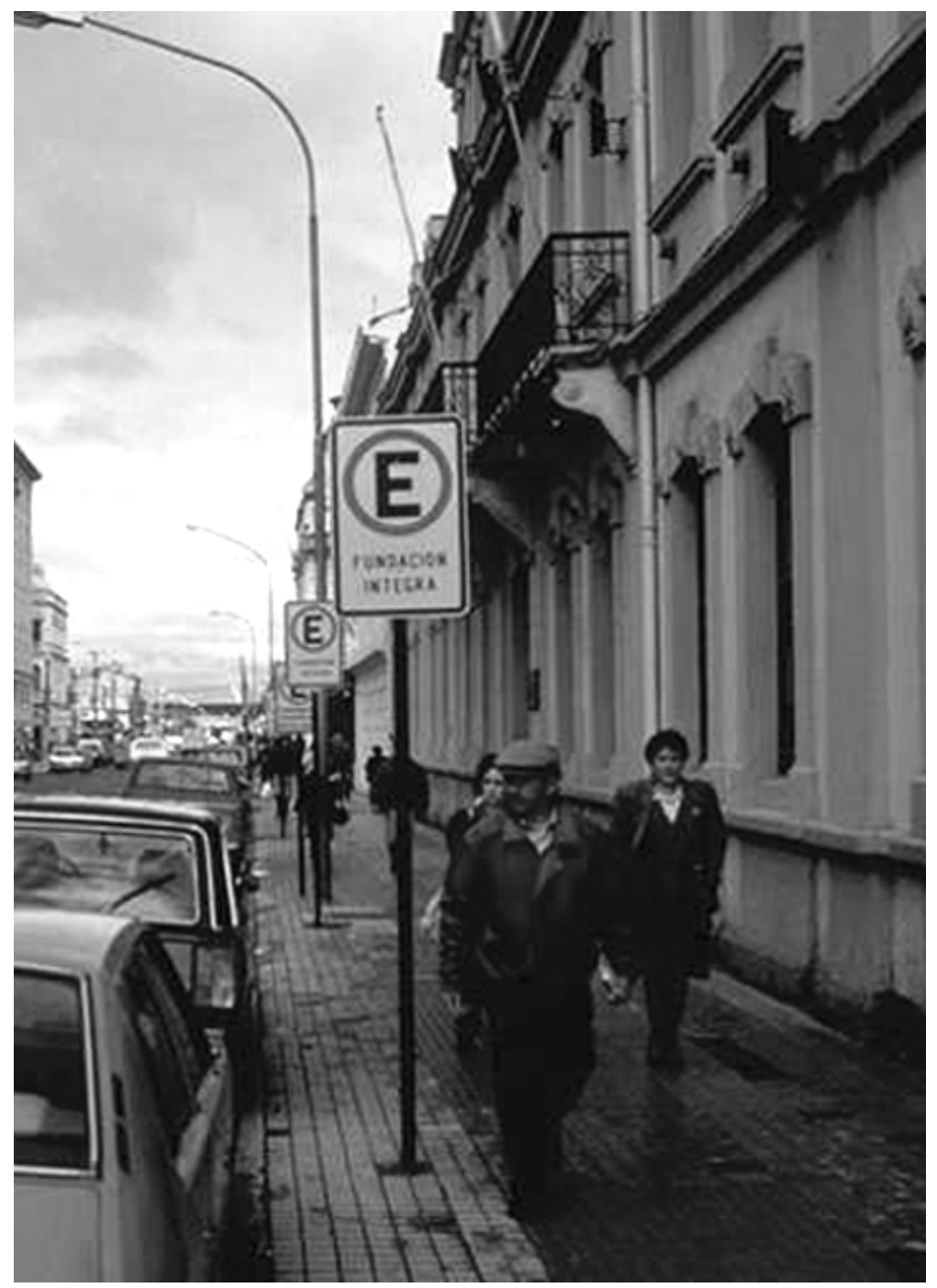




\title{
Resumen
}

En los últimos años las rentabilidades de los Fondos de Pensiones han bajado considerablemente, e incluso, en algunas oportunidades, éstas han sido negativas (en términos reales). Dada la obligatoriedad para un trabajador dependiente de ingresar a una Administradora de Fondos de Pensiones (AFP), en este trabajo se quiso analizar la combinación rentabilidad/ riesgo asumida por los afiliados.

Si bien los resultados de este trabajo señalan que, en general, la combinación rentabilidad/riesgo no es distinta a lo que la teoría predice, también es cierto la necesidad urgente de implementar los multifondos, para permitir que los distintos afiliados elijan los perfiles de rentabilidad/riesgo de acuerdo a sus preferencias.

Palabras clave: Riesgo, AFP, Multifondos, Previsión, Afiliados

\begin{abstract}
In the last years the interests of the pension funds have considerably gone down and sometimes it has been negative (in real terms). Due to the obligation of each worker to use a pension fund company, this research will analyze the combination of interest/risk to the affiliated workers.

Although the results of this research shows, in general terms, that the relation between interest/risk is not different from the theory, but in fact necessary to implement multi-funds, allows the affiliates to choose their profile of interest/risk.
\end{abstract}

Key words: Risk, Pension Fund, Multi-funds, Interest-risk, Affiliates 


Carlos Díaz Contreras
Freddy Higuera Cartes

\section{Fondos de pensiones: riesgo y rentabilidad; un análisis al caso chileno}

\section{Introducción}

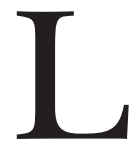

as Administradoras de Fondos de Pensiones (AFP) son Sociedades Anónimas que tienen como objeto exclusivo administrar un Fondo de Pensión, otorgar y administrar las prestaciones y los beneficios que establece la ley, como: recaudar las cotizaciones previsionales, mantener al día las cuentas individuales de los afiliados, invertir los recursos del Fondo y otorgar las pensiones a los beneficiarios.

El actual Sistema de Pensiones chileno se basa en la capitalización individual, esto significa que cada trabajador afiliado al sistema efectúa mensualmente un aporte previsional del $10 \%$ de su remuneración, el tope imponible es de $60 \mathrm{UF}^{1}$. Este aporte es acumulado en la cuenta de capitalización individual, la cual aumenta de acuerdo a los aportes que realiza el trabajador y a la rentabilidad que obtiene con la inversión de sus fondos. Además, cada trabajador debe cance- 
lar las comisiones que cobra la AFP como retribución por los servicios que presta. Actualmente cada AFP puede administrar sólo un Fondo de Pensiones.

Esta industria se inició el año 1981 con 11 AFP. En 1994, la industria alcanzó su mayor número, llegó a un total de 22 AFP. Hoy día, producto de fusiones, tan sólo hay $8 \mathrm{AFP}^{2}$. El objetivo de este trabajo es analizar el riesgo y la rentabilidad de los Fondos de Pensiones en relación con el riesgo y la rentabilidad obtenida por los accionistas de las AFP, asimismo, comparar el riesgo y la rentabilidad de otros tipos de fondos, como son los Fondos Mutuos de Renta Variable y los Fondos Mutuos de Renta Fija. Si los Fondos de Pensiones han obtenido menores rentabilidades o mayores riesgos que otras alternativas equivalentes, con mayor razón se justificaría la creación de los multifondos, tal como lo han sugerido distintos analistas económicos, de modo que cada trabajador pueda elegir la mejor combinación de riesgo y rentabilidad que desea asumir.

\section{Antecedentes}

Existe separación e independencia total entre el patrimonio de la AFP y el Fondo de Pensiones que ella administra, por lo que se llevan contabilidades separadas. El único flujo de dinero que existe desde el Fondo de Pensiones a la AFP corresponde a las comisiones. El valor del Fondo de Pensiones se expresa en cuotas de igual valor y características.

Los recursos de los Fondos de Pensiones sólo pueden invertirse en aquellos instrumentos que están expresamente autorizados por la Ley de Fondos de Pensiones. Actualmente, la norma citada autoriza la inversión en títulos estatales, instrumentos emitidos por instituciones financieras, bonos de empresas, bonos de empresas canjeables por acciones, acciones de sociedades anónimas inmobiliarias abiertas, cuotas de fondos de inversión, efectos de comercio emitidos por empresas e instrumentos extranjeros. Para cada uno de ellos existen límites mínimos y máximos, tanto en forma individual como por grupos.

De acuerdo con la Superintendencia de Administradoras de Fondos de Pensiones ${ }^{3}$, el Sistema de Pensiones chileno ha acumulado 
un $11 \%$ de rentabilidad promedio anual real desde sus inicios, lo que para muchos economistas y entendidos en la materia no es suficiente, pudiendo éste presentar una mayor rentabilidad. Más aún, esa tasa se ha venido reduciendo considerablemente, pues en el año 1998 el sistema finalizó con una caída de $1.1 \%$ real; fue la segunda oportunidad en la que el fondo de pensiones concluyó con un saldo negativo, pues en 1995 culminó con un $-2.5 \%$.

Incluso, se puede comprobar que en los últimos cuatro años el sistema acumula un retorno promedio de $1.15 \%$ real, rentabilidad muy inferior a la que presentan los papeles de renta fija, tanto de largo como de corto plazo. De hecho, en el año 1998 los depósitos a plazo a 30 días, si se hubiesen renovado periódicamente, habrían rentado sobre UF+7\%, mientras que las Administradoras de Fondos para la Vivienda (AFV) creadas recientemente, exhibieron una ganancia superior a $7 \%$ real $^{5}$.

Debido a lo anterior, se hace urgente que el sistema privado de pensiones recupere la credibilidad entre los cotizantes, para lo cual se requiere de una serie de reformas, las que fundamentalmente apunten a reducir los costos y a mejorar la relación riesgo-retorno, relación que se ve limitada por las restricciones actuales en materia de límites de inversión.

Se debe tener en cuenta que la rentabilidad histórica que han obtenido los Fondos de Pensiones no es aplicable a todas las personas, pues más de un $10 \%$ de los cotizantes del sistema (324,109 personas) ingresó en los últimos cuatro años, con lo cual han percibido una rentabilidad de apenas $1.15 \%$, perdiendo con este ahorro forzoso, la oportunidad de haber logrado retornos superiores en el sistema financiero ${ }^{6}$. Entonces, no resulta extraño que surjan voces que soliciten la posibilidad de que cada persona deposite sus fondos para pensionarse, en la alternativa financiera que estime más conveniente. Como una solución parcial a este problema, a partir del primer semestre del año 2000, entró en vigencia el Segundo Fondo de Pensiones (de Renta Fija). La creación del Fondo de Pensiones Tipo 2, como lo denomina la ley, responde a la conveniencia de ofrecer a los afiliados pensionados y próximos a pensionarse una alternativa de fondo con una rentabilidad menos fluctuante, para prevenir que variaciones eventuales pronunciadas en la rentabilidad de sus aho- 
rros previsionales, en los últimos años de cotización, puedan afectar la calidad de su pensión al momento de jubilarse. Por esto se concretó la creación de este fondo conformado sólo por títulos de renta fija de un plazo promedio acotado, lo que significa una rentabilidad más estable considerando que aproximadamente un $85 \%$ de la volatilidad del retorno del Fondo de Pensiones actual corresponde a los títulos de renta variable.

Los recursos del Segundo Fondo de Pensiones se invierten sólo en títulos de renta fija nacional o extranjera, con un plazo promedio que no puede ser inferior a dos años y medio ni superior a cuatro años, con el fin de reducir el riesgo de tasa de interés del portafolio de los Fondos de Pensiones. Este Fondo Tipo 2 debe cumplir con los mismos requisitos de Rentabilidad Mínima y Encaje que el Fondo Tipo 1.

Los afiliados que pueden incorporarse a este fondo son: hombres de 55 o más años de edad, mujeres de 50 o más años de edad y afiliados declarados inválidos mediante un primer dictamen.

\section{Multifondos}

La creación de un Tercer Fondo de Pensiones, que invierta sólo en instrumentos variables, será materia de un nuevo proyecto de ley, en todo caso, la idea de este Tercer Fondo es que el público pueda optar voluntariamente por él, con plena conciencia de que éste tendrá un nivel de riesgo aún mayor, porque se basará en instrumentos de variabilidad muy alta, que podrían ser nacionales o extranjeros.

El economista de Econsult, José Ramón Valente ${ }^{7}$, propone la creación de cinco tipos de fondos, segmentados por los distintos requerimientos que tienen los afiliados de acuerdo con sus edades, así podrían establecerse plazos de inversión de 1, 5, 10, 20 y 30 años, según el tiempo que le reste al afiliado para pensionarse.

\section{Rentabilidad-Riesgo}

La ley protege al trabajador de los riesgos derivados de un desempeño ineficiente de su AFP en la inversión del fondo, exigiéndose a ésta una rentabilidad mínima respecto del promedio del total de los fondos que manejan las Administradoras. Cada mes las AFP's son 
responsables de que la rentabilidad real de los últimos 12 meses del Fondo de Pensiones que administran no sea menor a la que resulte inferior entre la rentabilidad real de los últimos 12 meses, promedio de todos los Fondos, menos 2 puntos porcentuales, y el 50 por ciento de la rentabilidad real de los últimos 12 meses, promedio de todos los Fondos de Pensiones. Este sistema de cálculo de la rentabilidad mínima ha terminado por inducir a la concentración del sistema, ya que la AFP más grande, marca el promedio de rentabilidad. Con ello se estimula la imitación de las estrategias de inversión, desincentivando la competencia. A esta situación se le conoce como "efecto manada", para lo cual algunos especialistas proponen establecer una rentabilidad mínima, calculada sobre bases distintas al promedio del sistema y considerando una rentabilidad de más largo plazo.

\section{Cuotas de los Fondos de Pensiones}

El Fondo de Pensiones se expresa en "cuotas". La cuota es una unidad de medida definida por cada AFP, en forma independiente al empezar a funcionar, y expresa el valor del Fondo de Pensiones en unidades de igual monto y características.

El valor de la cuota de cada AFP variará diariamente, de acuerdo con las ganancias o pérdidas que tenga el fondo en sus inversiones. Los instrumentos con los que la ley permite invertir en este fondo se revalorizan cada día al valor de mercado, y el valor cuota se obtiene dividiendo el monto en pesos del fondo de pensiones por el número de cuotas que lo forman.

La rentabilidad de la cuota es el reflejo exclusivo de la ganancia obtenida por las inversiones que realiza la AFP y, por tanto, no depende ni del saldo acumulado ni de las comisiones cobradas.

\section{Fondos Mutuos}

Un Fondo Mutuo es un patrimonio integrado por aportes de personas naturales y jurídicas, denominadas partícipes, para su inversión en instrumentos financieros, tales como: acciones, cuotas de fondos de inversión, bonos, pagarés y letras hipotecarias, entre otros. Este patrimonio es administrado por una Sociedad Anónima, por 
cuenta y riesgo de los partícipes, quienes tienen derecho a rescatar, parcial o totalmente, sus aportes en cualquier momento, sujeto a las condiciones pactadas.

En Chile, existen tres tipos de fondos en los cuales se puede invertir:

- Fondos mutuos de inversión en instrumentos de renta fija de corto plazo que invierten en títulos estatales, depósitos y pagarés bancarios y bonos bancarios o de empresas. Estos fondos no pueden invertir más de $10 \%$ del valor del activo del fondo en instrumentos con un vencimiento mayor a 120 días.

- Fondos mutuos de inversión en instrumentos de renta fija de mediano y largo plazo que invierten en títulos estatales, depósitos, y pagarés y bonos bancarios o de empresas. Estos fondos no tienen restricciones para invertir el total de su activo en instrumentos de corto, mediano y largo plazo.

- Fondos mutuos de inversión en instrumentos de renta variable, los cuales pueden invertir en acciones, cuotas de fondos de inversión y títulos de renta fija de corto, mediano y largo plazo.

La inversión puede ser realizada en Chile y en el extranjero, según lo defina el propio Fondo Mutuo en su política de inversiones, pudiendo llegar en su cartera hasta a un $100 \%$ de sus activos en títulos extranjeros.

Todos los aportes se expresan en cuotas, que en su totalidad representan el valor neto del Fondo. La cuota es la unidad de la medida en que está dividido el patrimonio del Fondo. El monto de ellas varía a diario de acuerdo con el resultado de las inversiones y representan el grado de propiedad de los partícipes dentro del patrimonio del Fondo.

\section{Prueba para evaluar riesgo y rentabilidad}

\section{Prueba de Diferencias de Medias}

Para probar el comportamiento de las rentabilidades de los Fondos de Pensiones, se usará una prueba de diferencias de medias. 


$$
\frac{\overline{x_{1}}-\overline{x_{2}}}{\sqrt{\left(\frac{1}{n_{1}}+\frac{1}{n_{2}}\right)\left(\frac{S_{1}+S_{2}}{n_{1}+n_{2}-2}\right)}} \geq t\left(\alpha, n_{1}+n_{2}-2\right)
$$

Donde:

$S_{i}$ : suma de los cuadrados de la muestra i.

$\overline{X_{i}}$ : media de la muestra $\mathrm{i}$.

$\alpha \quad$ : nivel de significancia (5\%).

$S=\sum(x-\bar{x})^{2}$

\section{Prueba de Varianzas}

Para probar el comportamiento de riesgo de los Fondos de Pensiones, se usará una prueba de varianzas.

$$
\frac{V_{1}^{2}}{V_{2}^{2}} \geq F\left(\alpha, n_{1}-1, n_{2}-1\right)
$$

Donde:

$V_{i}^{2}$ : nivel de significancia

$\alpha \quad$ : nivel de significancia (5\%).

Análisis comparativo entre los fondos de pensiones y las AFP

Se estudiarán las cinco AFP que han cotizado en la Bolsa de Valores, por un período de ocho años, las cuales son: Cuprum, Habitat, Protección, Provida y Santa María.

Se realizará una comparación entre el Fondo de Pensiones que maneja cada AFP y sus resultados como Sociedad Anónima.

\section{Período}

Se analizará el período comprendido desde enero de 1991 hasta diciembre de 1998. Todos los datos (valores cuotas y precios de las acciones) están al último día hábil de cada mes. 


\section{Hipótesis de Rentabilidad}

La rentabilidad del Fondo de Pensiones es menor o igual a la rentabilidad de la AFP, es decir:

$\mathrm{H}_{\mathrm{o}}: \mu_{1, \mathrm{i}} \leq \mu \mathrm{m}_{2, \mathrm{i}}$

$\mathrm{H}_{1}: \mu_{1, \mathrm{i}}>\mu_{2, \mathrm{i}}$

Donde:

$\mu_{1, \mathrm{i}}$ : rentabilidad del valor cuota del Fondo de Pensiones i.

$\mu_{2, \mathrm{i}}$ : rentabilidad de las acciones de la Sociedad Anónima i (AFPi).

La región crítica se encuentra al lado derecho de la distribución $\mathrm{t}$ con $\left(n_{i}+n_{j}-2\right)$ grados de libertad.

\section{Hipótesis de Riesgo}

El riesgo del Fondo de Pensiones es menor o igual al riesgo de la AFP, es decir:

$H_{0}: \sigma_{1, i}{ }^{2} \leq \sigma_{2, i}{ }^{2}$

$\mathrm{H}_{1}: \sigma_{1, \mathrm{i}}{ }^{2}>\sigma_{2, \mathrm{i}}{ }^{2}$

Donde:

$\sigma_{1, i}{ }^{2}$ : riesgo del valor cuota del Fondo de Pensiones i.

$\sigma_{2, i}{ }^{2}$ : riesgo de las acciones de la Sociedad Anónima i (AFPi).

La región crítica se encuentra al lado derecho de la distribución F, con $\left(n_{i}-1, n_{j}-1\right)$ grados de libertad.

\section{Resultados Pruebas de Medias}

Los resultados de las Pruebas de Medias se muestran en la Tabla 1. 
Tabla 1

Pruebas de Medias

\begin{tabular}{|clcccc|}
\hline Período & AFP & Valor Observado & & Valor Tabla & $\mathbf{H}_{\mathbf{0}}$ \\
$1991-1998$ & Cuprum & -1.5520 & $<$ & 1.653 & No se rechaza \\
$1991-1998$ & Hábitat & -0.5510 & $<$ & 1.653 & No se rechaza \\
$1991-1998$ & Protección & -1.4180 & $<$ & 1.653 & No se rechaza \\
$1991-1998$ & Provida & -0.3809 & $<$ & 1.653 & No se rechaza \\
$1991-1998$ & Sta. María & -0.3419 & $<$ & 1.653 & No se rechaza \\
\hline
\end{tabular}

$\mathbf{H}_{0}=$ rentabilidad Fondo de Pensiones $<$ rentabilidad dueños AFP

En todos los casos las pruebas no se rechazaron, es decir, estadísticamente, con un $95 \%$ de confianza, la rentabilidad de las AFP siempre fue menor o igual que la rentabilidad obtenida por los dueños de las AFP.

\section{Resultados Pruebas de Varianzas}

Los resultados de las Pruebas de Varianzas se muestran en la Tabla 2.

\section{Tabla 2}

Pruebas de Varianzas

\begin{tabular}{|c|c|c|c|c|c|}
\hline Período & AFP & Valor Observado & & Valor Tabla & $\mathbf{H}_{0}$ \\
\hline $1991-1998$ & Cuprum & 0.0199 & $<$ & 1.404 & No se rechaza \\
\hline $1991-1998$ & Hábitat & 0.0802 & $<$ & 1.404 & No se rechaza \\
\hline $1991-1998$ & Protección & 0.0182 & $<$ & 1.404 & No se rechaza \\
\hline $1991-1998$ & Provida & 0.0335 & $<$ & 1.404 & No se rechaza \\
\hline $1991-1998$ & Sta. María & 0.0290 & $<$ & 1.404 & No se rechaza \\
\hline
\end{tabular}

$\mathbf{H}_{0}=$ volatilidad Fondo de Pensiones $<$ volatilidad dueños AFP

En la totalidad de los casos, las pruebas de varianzas no se rechazaron, por lo cual se concluye, que con $95 \%$ de confianza el riesgo asumido por los afiliados siempre fue menor o igual que el riesgo que asumieron los dueños de las AFP. 


\section{Conclusión}

Para el período analizado, siempre los afiliados a las AFP obtuvieron una rentabilidad menor o igual que los dueños de las AFP, pero en compensación a ello, en todos los casos siempre asumieron un riesgo menor o igual que los dueños.

\section{Análisis comparativo entre los fondos de pensiones y los fondos mutuos}

Para realizar el análisis de riesgo-rentabilidad de los Fondos Mutuos (FM), se eligieron cuatro tipos de fondos distintos los cuales debían estar vigentes, al menos, desde 1991, de manera que el período de análisis coincidiera con el analizado para los Fondos de Pensiones. Para este análisis no se consideraron los Fondos Mutuos de Renta Fija de Corto Plazo, debido a que las inversiones realizadas por las Administradoras de Fondos de Pensiones están orientadas al largo plazo. Así, se escogieron dos fondos de renta variable y dos fondos de renta fija de mediano y largo plazo, de distintas instituciones financieras. Éstas son:

\section{Fondos Mutuos de Renta Fija de Mediano y Largo Plazo}

- FM Inversión y Ahorro del Banco Bice: sus objetivos de inversión es el ahorro en instrumentos de renta fija nacionales, con liquidez mayor que los instrumentos tradicionales de ahorro de largo plazo. Está dirigido a personas naturales o jurídicas.

- FM Proyección del Banco Citicorp: este Fondo está orientado hacia las personas que buscan ahorrar o invertir en el mediano y largo plazo, aprovechando las oportunidades de una cartera diversificada, compuesta por bonos de empresas e instrumentos emitidos o garantizados por el Estado de Chile y los principales bancos comerciales del país. 


\section{Fondos Mutuos de Renta Variable}

- FM Santander Acciones del Banco Santander: este instrumento de inversión de largo plazo, está orientado a inversionistas y ahorristas que persiguen los beneficios de la rentabilidad de una cartera diversificada, compuesta fundamentalmente por acciones de las principales empresas del país, seleccionadas y analizadas por especialistas del banco.

- FM Banchile Capitalisa del Banco de Chile: este fondo invierte en una cartera diversificada de acciones de transacción bursátil y otros valores de oferta pública autorizados por la Superintendencia de Valores y Seguros.

\section{Datos}

Se consideró el valor cuota del último día hábil que se publica en cada mes, a partir de enero de 1991 hasta diciembre de 1998. Los valores de las cuotas están ajustados por el Índice de Precios al Consumidor (IPC), a diciembre de 1998.

\section{Planteamiento de Hipótesis}

Se plantean las mismas hipótesis que en la sección anterior, con la diferencia que ahora la rentabilidad/riesgo del Fondo de Pensiones se compara con la rentabilidad/riesgo de los Fondos Mutuos.

\section{Resultados Pruebas de Medias para los Fondos Mutuos de Renta Fija}

Al comparar las rentabilidades de los Fondos de Pensiones con la del Fondo Mutuo de Renta Fija Proyección del Banco Citicorp (Tablas 3 y 4), una de las cinco AFP tuvo una rentabilidad menor o igual en el período de 8 años. En cambio, si se comparan con el Fondo Mutuo Inversión y Ahorro del Banco Bice, las AFP que obtuvieron una menor rentabilidad, aumentan a 3 para el mismo período. 


\section{Díaz Contreras / Higuera Cartes}

Tabla 3

Prueba de Media (Fondo de Pensiones v/s FM Proyección)

\begin{tabular}{|c|c|c|c|c|c|}
\hline Período & AFP & Valor Observado & & Valor Tabla & $\mathbf{H}_{0}$ \\
\hline $1991-1998$ & Cuprum & 1.8120 & $>$ & 1.653 & Se rechaza \\
\hline $1991-1998$ & Hábitat & 1.3650 & $\leq$ & 1.653 & No se rechaza \\
\hline $1991-1998$ & Protección & 1.8840 & $>$ & 1.653 & Se rechaza \\
\hline $1991-1998$ & Provida & 1.7880 & $>$ & 1.653 & Se rechaza \\
\hline $1991-1998$ & Sta. María & 1.7670 & $>$ & 1.653 & Se rechaza \\
\hline
\end{tabular}

$\mathbf{H}_{\mathbf{0}}=$ rentabilidad Fondo de Pensiones $£$ rentabilidad Fondo Mutuo de Renta Fija.

Tabla 4

Prueba de Media (Fondo de Pensiones v/s FM Inversión y Ahorro)

\begin{tabular}{|llllll|}
\hline Período AFP & Valor Observado & & \multicolumn{2}{l}{ Valor Tabla } & $\mathbf{H}_{\mathbf{0}}$ \\
$1991-1998$ & Cuprum & 1.6490 & $\leq$ & 1.653 & No se rechaza \\
$1991-1998$ & Hábitat & 1.1570 & $\leq$ & 1.653 & No se rechaza \\
$1991-1998$ & Protección & 1.7330 & $>$ & 1.653 & Se rechaza \\
$1991-1998$ & Provida & 1.6260 & $\leq$ & 1.653 & No se rechaza \\
$1991-1998$ & Sta. María & 1.5960 & $>$ & 1.653 & Se rechaza \\
\hline
\end{tabular}

$\mathbf{H}_{\mathbf{0}}=$ rentabilidad Fondo de Pensiones $£$ rentabilidad Fondo Mutuo de Renta Fija

\section{Resultados Pruebas de Medias para los Fondos Mutuos de Renta Variable}

Al comparar las rentabilidades de los Fondos de Pensiones con las rentabilidades de ambos Fondos Mutuos de Renta Variable (ver Tablas 5 y 6), todas las AFP tuvieron una rentabilidad menor o igual (nunca la hipótesis nula se rechazó). 
Tabla 5

Prueba de Media (Fondo de Pensiones v/s FM Acciones)

\begin{tabular}{|clcccc|}
\hline Período & AFP & \multicolumn{2}{c}{ Valor Observado } & & \multicolumn{2}{c|}{ Valor Tabla } & $\mathbf{H}_{\mathbf{0}}$ \\
$1991-1998$ & Cuprum & 0.4213 & $\leq$ & 1.653 & No se rechaza \\
$1991-1998$ & Hábitat & 0.4539 & $\leq$ & 1.653 & No se rechaza \\
$1991-1998$ & Protección & 0.4559 & $\leq$ & 1.653 & No se rechaza \\
$1991-1998$ & Provida & 0.3959 & $\leq$ & 1.653 & No se rechaza \\
$1991-1998$ & Sta. María & 0.4016 & $\leq$ & 1.653 & No se rechaza \\
\hline
\end{tabular}

$\mathbf{H}_{0}=$ rentabilidad Fondo de Pensiones $\leq$ rentabilidad Fondo Mutuo de Renta Variable.

Tabla 6

Prueba de Media (Fondo de Pensiones v/s FM Capitalisa)

\begin{tabular}{|llllll|}
\hline Período AFP & \multicolumn{2}{l}{ Valor Observado } & \multicolumn{2}{l|}{ Valor Tabla } & $\mathbf{H}_{\mathbf{0}}$ \\
$1991-1998$ & Cuprum & 1.0570 & $\leq$ & 1.653 & No se rechaza \\
$1991-1998$ & Hábitat & 1.0380 & $\leq$ & 1.653 & No se rechaza \\
$1991-1998$ & Protección & 1.0920 & $\leq$ & 1.653 & No se rechaza \\
$1991-1998$ & Provida & 1.0330 & $\leq$ & 1.653 & No se rechaza \\
$1991-1998$ & Sta. María & 1.0370 & $\leq$ & 1.653 & No se rechaza \\
\hline
\end{tabular}

$\mathbf{H}_{0}=$ rentabilidad Fondo de Pensiones $\leq$ rentabilidad Fondo Mutuo de Renta Variable.

\section{Resultados Prueba de Varianza para los Fondos Mutuos de Renta Fija}

A pesar de que los Fondos de Pensiones no siempre alcanzaron una mayor rentabilidad que los Fondos Mutuos de Renta Fija (ver Tablas 7 y 8), siempre estuvieron sujetos a un riesgo mayor en el período 1991-1998. 
Díaz Contreras / Higuera Cartes

Tabla 7

Prueba de Varianza (Fondo de Pensiones v/s FM Proyección)

\begin{tabular}{|clcccc|}
\hline Período & AFP & \multicolumn{2}{c}{ Valor Observado } & Valor Tabla & $\mathbf{H}_{\mathbf{0}}$ \\
$1991-1998$ & Cuprum & 1.7880 & $>$ & 1.404 & Se rechaza \\
$1991-1998$ & Hábitat & 4.9290 & $>$ & 1.404 & Se rechaza \\
$1991-1998$ & Protección & 1.8460 & $>$ & 1.404 & Se rechaza \\
$1991-1998$ & Provida & 1.6450 & $>$ & 1.404 & Se rechaza \\
$1991-1998$ & Sta. María & 1.7670 & $>$ & 1.404 & Se rechaza \\
\hline
\end{tabular}

$\mathbf{H}_{\mathbf{0}}=$ volatilidad Fondo de Pensiones $\leq$ volatilidad Fondo Mutuo de Renta Fija.

Tabla 8

Prueba de Varianza (Fondo de Pensiones v/s FM Inversión y Ahorro)

\begin{tabular}{|clcccc|}
\hline Período & AFP & \multicolumn{2}{c}{ Valor Observado } & Valor Tabla & $\mathbf{H}_{\mathbf{0}}$ \\
$1991-1998$ & Cuprum & $9.5430 \quad>$ & 1.404 & Se rechaza \\
$1991-1998$ & Hábitat & 26.3100 & $>$ & 1.404 & Se rechaza \\
$1991-1998$ & Protección & $9.8510 \quad>$ & 1.404 & Se rechaza \\
$1991-1998$ & Provida & 8.7780 & $>$ & 1.404 & Se rechaza \\
$1991-1998$ & Sta. María & 20.4300 & $>$ & 1.404 & Se rechaza \\
\hline
\end{tabular}

$\mathbf{H}_{\mathbf{0}}=$ volatilidad Fondo de Pensiones $\leq$ volatilidad Fondo Mutuo de Renta Fija.

\section{Resultados Prueba de Varianza para los Fondos Mutuos de Renta Variable}

En este caso, los resultados son los esperados, es decir, el riesgo de los Fondos de Pensiones fue menor que el de los Fondos Mutuos de Renta Variable (ver Tabla 9 y 10). 
Tabla 9

Prueba de Varianza (Fondo de Pensiones v/s FM Acciones)

\begin{tabular}{|c|c|c|c|c|c|}
\hline Período & A.F.P & Valor Observado & & Valor Tabla & $\mathbf{H}_{0}$ \\
\hline $1991-1998$ & Cuprum & 0.1050 & $\leq$ & 1.404 & No se rechaza \\
\hline $1991-1998$ & Hábitat & 0.2894 & $\leq$ & 1.404 & No se rechaza \\
\hline $1991-1998$ & Protección & 0.1084 & $\leq$ & 1.404 & No se rechaza \\
\hline $1991-1998$ & Provida & 0.0966 & $\leq$ & 1.404 & No se rechaza \\
\hline $1991-1998$ & Sta. María & 0.1037 & $\leq$ & 1.404 & No se rechaza \\
\hline
\end{tabular}

$\mathbf{H}_{0}=$ volatilidad Fondo de Pensiones $\leq$ volatilidad Fondo Mutuo de Renta Variable.

Tabla 10

Prueba de Varianza (Fondo de Pensiones v/s FM Capitalisa)

\begin{tabular}{|llcccc|}
\hline Período & AFP & Valor Observado & & Valor Tabla & $\mathbf{H}_{\mathbf{0}}$ \\
$1991-1998$ & Cuprum & 0.1183 & $\leq$ & 1.404 & No se rechaza \\
$1991-1998$ & Hábitat & 0.3260 & $\leq$ & 1.404 & No se rechaza \\
$1991-1998$ & Protección & 0.1221 & $\leq$ & 1.404 & No se rechaza \\
$1991-1998$ & Próvida & 0.1088 & $\leq$ & 1.404 & No se rechaza \\
$1991-1998$ & Sta. María & 0.1169 & $\leq$ & 1.404 & No se rechaza \\
\hline
\end{tabular}

$\mathbf{H}_{0}=$ volatilidad Fondo de Pensiones $£$ volatilidad Fondo Mutuo de Renta Variable.

El comportamiento de los Fondos de Pensiones no siempre fue el esperado, ya que en algunas AFP, dichos Fondos de Pensiones han resultado menos rentables y más riesgosos que las alternativas que ofrecen los Fondos Mutuos de Renta Fija. En cambio, cuando esta comparación se hace con los Fondos Mutuos de Renta Variable, las combinaciones riesgo y rentabilidad obtenidas son las esperadas. 


\section{Conclusiones}

Las Administradoras de Fondos de Pensiones, en sus 19 años de funcionamiento, han alcanzado una rentabilidad promedio de un $11 \%$ anual. Si bien esta rentabilidad se podría considerar «alta», ya que es superior a la que se esperaba cuando se echó a andar el sistema, este promedio está fuertemente sustentado por las buenas rentabilidades de los primeros años. En los últimos años las rentabilidades han bajado considerablemente, e incluso, en algunas oportunidades, éstas han sido negativas en términos reales.

Dada la obligación de un trabajador dependiente de ingresar a una AFP, en este trabajo se quiso analizar la combinación rentabilidad/ riesgo asumida por los afiliados. Las principales conclusiones de este análisis son:

- Los accionistas de las AFP obtienen mayor rentabilidad que los afiliados a las AFP, pero también asumen mayor riesgo que estos últimos.

- Los afiliados a las AFP no siempre han obtenido mayor rentabilidad que los Fondos Mutuos de Renta Fija, pero siempre han asumido un riesgo mayor.

- Los afiliados a las AFP siempre han tenido menor rentabilidad que los Fondos Mutuos de Renta Variable, pero también han asumido un riesgo menor.

Si bien con los resultados anteriores se podría concluir que, en términos generales, la combinación rentabilidad/riesgo de los afiliados está acorde con lo esperado, también es cierto que un afiliado podría preferir optar por una rentabilidad esperada mayor aunque tenga que aumentar su riesgo, así como habrá otros que preferirán lo contrario, es decir, menos rentabilidad esperada, pero menor riesgo.

La creación del Segundo Fondo, que entró en vigencia, viene a solucionar una parte del problema (afiliados que deseen menor riesgo y que estén dispuestos a optar por una menor rentabilidad esperada), pero no satisface los requerimientos de muchos otros afiliados que preferirían que sus fondos se invirtieran mayoritariamente en 
instrumentos de renta variable, sobre todo afiliados jóvenes que recién se incorporan al sistema, a los que aún les quedan 30 o 40 años para jubilarse. Por lo tanto, la posibilidad de que existan multifondos con distintos perfiles de rentabilidad/riesgo, más que un deseo es una necesidad ${ }^{9}$. 


\section{Notas}

${ }^{1}$ U.F. = Unidad de Fomento. Al día 30 de noviembre de 1999 tiene un valor de $\$ 14.998$, y éste se va modificando diariamente de acuerdo a la variación experimentada por el Índice de Precios al Consumidor mensual (inflación mensual) de la siguiente manera:

- al inicio de cada mes se conoce la inflación del mes anterior.

- con este dato se calcula cuánto debe aumentar el valor de la Unidad de Fomento en el presente mes.

- esa variación se divide por el número de días que tiene el mes y con ello se tieneuna variación diaria.

2 Serie de Estudios, Asociación de AFP, № 2, Diciembre 1999.

3 Diario Estrategia, viernes, 24 de septiembre de 1999, pág. 3.

${ }^{4}$ Diario El Mercurio, jueves 11 de febrero de 1999.

5 Diario Estrategia, lunes, 8 de febrero de 1999, pág. 16.

6 Diario Estrategia, lunes, 8 de febrero de 1999, pág. 16.

7 Diario Estrategia, lunes, 26 de julio de 1999, pág. 23.

8 "Regulación de Inversiones de las AFP: ¿es necesario avanzar hacia fondos múltiples?”, pág. 10. Dirección en Internet: http://www.afp-.cl/ congreso/papers/parte1.pdf

${ }^{9}$ A partir del 1 de agosto de 2002, en Chile se crearon los multifondos. Ahora, cada AFP administra cinco fondos que se diferencian por el porcentaje de recursos que pueden destinar a la compra de instrumentos de renta variable y por su exposición al tipo de cambio (dólar). Estos fondos son:

Fondo A: $40 \%$ a 80 de Renta variable.

Fondo B: $25 \%$ a $60 \%$ de Renta Variable.

Fondo C: $15 \%$ a $40 \%$ de Renta Variable (similar al Fondo Tipo 1 existente antes de esa fecha).

Fondo D: $5 \%$ a $20 \%$ de Renta Variable.

Fondo E: 0\% de Renta Variable (similar al Fondo Tipo 2 creado en el primer semestre de 2000). 


\section{Bibliografía}

AFP Cuprum. "Documento Informativo”. Boletines $N^{o} 1$ al $N^{o} 27$.

Bice Fondos Mutuos S.A. "Documentos Fondos Mutuos”. Boletín, abril de 1999.

Caballero, F., Gonzáles, R., Pareja, A. y Triguero F. Métodos Matemáticos para la Economía. Editorial Mc Graw-Hill, Primera Edición, 1992.

Flores, A. y Núñez, F. "Análisis de la Rentabilidad Histórica de los Fondos de Pensiones y su Proyección”. Tesis de Grado para optar al grado académico de Licenciado en Ciencias de la Administración de Empresas y título profesional de Ingeniero Comercial, Universidad Católica del Norte, Chile, diciembre de 1997.

Gonzáles, C. Reforma Previsional DL No 3500, Ediciones Publiley, Chile, 1999.

Kaufman, A. y Cruon, R. La Programación Dinámica. Editorial Continental S.A. España, Primera Edición, 1967.

Kazmier, L. y Díaz, A. Estadística Aplicada a la Economía. Editorial Mc Graw-Hill, Segunda Edición, 1995.

Kazmier, L. y Díaz, A. Estadística Aplicada a la Administración y a la Economia. Serie Shaum, ed. Mc Graw-Hill, Segunda Edición, febrero de 1995.

Superintendencia de Administradoras de Fondos de Pensiones. "Evolución del Sistema Chileno de Pensiones № 3 (1981-1997)”. Publicación S.A.F.P., marzo de 1998.

Saldías, R. "Una nota sobre la cartera del Fondo de Pensiones". Revista Economía y Administración, № 45, diciembre de 1995.

Tarziján, J. "Reflexiones sobre la Rentabilidad Mínima exigida a las AFP". Revista Administración y Economía Universidad Católica de Chile, verano 1995 , № 20, págs. 23-26.

Walker, E. "Reflexiones en torno a políticas de inversión adecuadas para las Administradoras de Fondos de Pensiones". Cuadernos de Economía Universidad Católica de Chile, diciembre de 1991, № 85, págs. 359-384. 
